\title{
Motility-related protein-I (MRP-I/CD9) expression can predict disease-free survival in patients with squamous cell carcinoma of the head and neck
}

\author{
P Mhawech", , P Dulguerov' ${ }^{2}$ E Tschanz', C Verdan', C Ares ${ }^{3}$ and AS Allal ${ }^{3}$ \\ 'Department of Pathology, Geneva University Hospital, CMU, Michel-Servet, 1, Geneva 1211, 4, Switzerland; '2Division of Head and Neck Surgery, \\ Geneva University Hospital, CMU, Michel-Servet, I, Geneva 1211, 4, Switzerland; ${ }^{3}$ Division of Radiation Oncology, Geneva University Hospital, CMU, \\ Michel-Servet, I, Geneva 1211, 4, Switzerland
}

\begin{abstract}
CD9 is a transmembrane protein that has been implicated in cell adhesion, motility and proliferation, and numerous studies have demonstrated the prognostic value of its expression in different solid tumours. The purpose of this study is to determine the predictive value of CD9 in squamous cell carcinoma (SCC) of the head and neck. A total of I53 cases were examined for CD9 expression using immunohistochemistry applied on formalin-fixed, paraffin-embedded tissue. Cases were stratified in two categories depending on CD9 expression, as positive ( $\geqslant 50 \%$ positive cells) or reduced ( $<50 \%)$. In all, I08 cases were positive for CD9 (85 cases with membranous, and 23 with both membranous and cytoplasmic staining) and 45 reduced expression. Reduced CD9 expression was significantly associated with high grade $(P=0.0007)$ and lower disease-free survival $(D F S)(P=0.017)$. The latter retained its significance in the multivariate analysis. When the 23 cases with both membranous and cytoplasmic patterns were studied as a separate subgroup, there were significant associations between CD9 expression and tumour grade $(P=0.025)(95 \% \mathrm{Cl} I \mathrm{I}-68)$, tumour stage $(P=0.08)(95 \% \mathrm{Cl} 3.5-86)$ and the occurrence of any failure $(P=0.083)(95 \% \mathrm{Cl}-1.7-57)$. Immunohistochemical CD9 expression proved to be an independent prognostic factor in SCC of the head and neck, and it may detect patients at a high risk of recurrence. In addition, the cytoplasmic pattern seems to have an even more significant value. However, this finding is limited to the small number of cases with this pattern.

British Journal of Cancer (2004) 90, 47I-475. doi:I0.1038/sj.bjc.660I542 www.bjcancer.com

(c) 2004 Cancer Research UK
\end{abstract}

Keywords: CD9 expression; IHC; SCC of head and neck; DFS

Despite the important advances made in the management of squamous cell carcinoma (SCC) of the head and neck, patients still have poor prognosis with a 5-year disease-free survival (DFS) of about $50 \%$ (Landis et al, 1999). The prognostic factors are mostly confined to the histopathologic and clinical parameters such as grade, stage, pattern of invasion, location and lymph node metastasis (Crissman et al, 1984; Pera et al, 1986; Wiernik et al, 1991; DeStefani et al, 1997). Recently, numerous molecular markers such as cell cycle regulators, cell adhesion proteins, oncogenes and tumour-suppressor genes have been investigated in SCC of the head and neck, and some of these markers have shown promising results for future use (Schoelch et al, 1999; Grabenbauer et al, 2000; Pukkila et al, 2001; Khademi et al, 2002; Khan et al, 2002; Liu et al, 2003).

Disruption of cell adhesion and alteration of cell motility play an important role in cancer cell invasion and metastasis. Previously, CD9 was reported to be of prognostic significance in adenocarci-

\footnotetext{
*Correspondence: P Mhawech;

E-mail: Paulette.Mhawech-Fauceglia@hcuge.ch

Received 26 June 2003; revised 9 October 2003; accepted 5 November 2003
}

noma of the lung, colon, breast, pancreas and SCC of the oesophagus and the oral cavity. Motility-related protein (MRP-1)/CD9 is a member of the transmembrane 4 superfamily (TM4SF) which is involved in cell growth, adhesion and motility. Of the 20 known members, five - MRP1/CD9, ME491/ CD63, KAI1/CD82, CD151, CD81 - may be implicated in cell migration, proliferation and tumour cell metastasis (Miyake et al, 1991; Ikeyama et al, 1993). The motility-related protein/CD9 is so far the best characterised of the TM4SF members. It is located on chromosome 12 (12p13) and is widely distributed among all cell types. CD9 is capable of interacting with other transmembrane proteins such as integrins and other tetraspanins to form a complex, which facilitates cell adhesion, motility and signalling (Hemler et al, 1996). Thus, CD9 appears to have an important role in inhibiting cell motility in numerous neoplastic cell lines (Miyake et al, 1991).

Studies evaluating the expression of this CD9 protein in SCC of the head and neck are nonexistent, save only for one study, and it was limited to the SCC of the oral cavity (Kusukawa et al, 2001). Thus, the aim of this study is to assess the utility of the CD9 protein expression in predicting DFS of patients with SCC arising in the head and neck after treatment with radiotherapy (RT) with or without chemotherapy. 


\section{MATERIALS AND METHODS}

\section{Patients population}

A retrospective study covering 7 years $(1992-1999)$ was conducted. The criteria for inclusion were patients with no prior treatment, histologic diagnosis performed in our department and adequate material for analysis. Tumours with a nasopharyngeal origin were excluded from the study. From 201 registered patients, 153 met our criteria for inclusion. The patient and tumour characteristics are summarised in Table 1. After the initial diagnosis, patients were treated with radical RT with or without chemotherapy. All patients were regularly followed up by the otolaryngologist, and the radio-oncologist. Therapy modality and follow-up data were retrieved from the registry at the Division of Radiation Oncology. The median follow-up for the surviving patients was 65 months (range 14-123).

\section{Treatment}

All patients received the same accelerated RT schedule using concomitant boost technique. The latter has been described previously in detail (Allal et al, 1999). The planned total dose was $69.9 \mathrm{~Gy}$, delivered in 41 fractions over a period of 38 days. The basic course was given to a total dose of $50.4 \mathrm{~Gy}$ over 5.5 weeks. The boost to the initial sites of macroscopic tumour involvement consisted of $19.5 \mathrm{~Gy}$, and was given as a second daily fraction, starting the last day of the second week of the basic treatment. According to our institutional policy, 20 patients (13\%) underwent a planned neck dissection prior to RT, either radical or modified radical, while one patient had simple excisions of lymph node metastases. Otherwise, surgery was reserved for salvage of locoregional failures.

Chemotherapy was given to 38 patients (25\%), usually for patients presenting with $\mathrm{T} 3-4$ or $\mathrm{N} 2-3$ tumours, if their medical condition was judged fit enough to tolerate multimodality treatment. Except for two patients who received induction chemotherapy, all patients received one or more cycles of cisplatin and 5-FU-based chemotherapy, concomitantly with RT.

\section{Immunohistochemistry}

The tissue analysed consisted of initial (pretreatment) biopsies. The original diagnosis was reviewed by two pathologists (PM, ET) and the histologic grade was assessed using the WHO system. Immunohistochemistry (IHC) was performed on paraffin-embedded tissues. Tissue sections were deparaffinised with xylene and washed with ethanol. For immunostaining enhancement, pretreatment by microwave oven in $0.01 \mathrm{M}$ citrate buffer $(\mathrm{pH} 6.0)$ at

Table I Patients and tumours characteristics

\begin{tabular}{lc}
\hline Median age (years) (range) & $59(35-90)$ \\
Sex M: F & I22/3I \\
Tumour sublocation & \\
Oral cavity & 8 \\
Oropharynx & 93 \\
Hypopharynx & 25 \\
Larynx & 27 \\
TN classification and stages (UICC 1997) & \\
TI -2 & 64 \\
T3-4 & 89 \\
NO & 72 \\
N+ & 81 \\
Stage I-II & 35 \\
Stage III-IV & 118 \\
Histology grading & \\
Well differentiated (GI) & 64 \\
Moderately differentiated (G2) & 66 \\
Poorly differentiated (G3) & 23 \\
\hline
\end{tabular}

$98^{\circ} \mathrm{C}$ for $30 \mathrm{~min}$ was done. Sections were incubated with a monoclonal anti-CD9 antibody (Novacastra, Newcastle, UK), diluted at $1: 20$. Endogenous peroxidase was blocked with $0.3 \%$ hydrogen peroxidase for $5 \mathrm{~min}$. Sections were then incubated with mouse Envision horseradish peroxidase (HRP) for $30 \mathrm{~min}$. IHC was performed using the automated stainer Dako 'Autostainer' (Dako, Copenhagen, Denmark). These incubations were performed at room temperature, and sections were washed by Tris buffer saline between incubations. Diaminobenzidine complex was used as chromogen. Tissue sections from cases of fibroadenoma were taken as positive controls. Staining of normal squamous epithelium with anti-CD9 showed strong membranous positivity (Figure 1). In negative controls, a normal goat serum was used in place of the primary antibody, resulting in a lack of detectable staining. Evaluation of the IHC was done twice by one pathologist $(\mathrm{PM})$ at 2 weeks interval. All the tissue on the slide was scanned for CD9 expression, and the average was calculated for the entire slide. Samples for CD9 expression were stratified into two categories, depending on the percentage of CD9-positive cells of any pattern (cytoplsamic/membranous). Cases showing $\geqslant 50 \%$ CD9-positive tumour cells were classified as 'positive', while cases with $<50 \%$ stained tumour cells were considered as 'reduced' cases.

\section{Statistical analysis}

The Mann-Whitney and Kruskal-Wallis tests were used to compare the CD9 median values of the different subgroups. The actuarial overall and DFS rates were calculated using the KaplanMeier method. For comparison between curves, the log-rank test was used. Multivariate analyses based on Cox proportional hazards standard model were used to identify the most significant factors related to outcomes. $P$-values of 0.05 or less were considered significant. All analysis was performed with the StatView V 5.0.1 software.

\section{RESULTS}

\section{Overall results}

At last follow-up, 58 patients were alive, and 95 had died. In all, 56 patients presented with one or more events. A total of 33 patients presented with persistent or recurrent local disease, 15 with

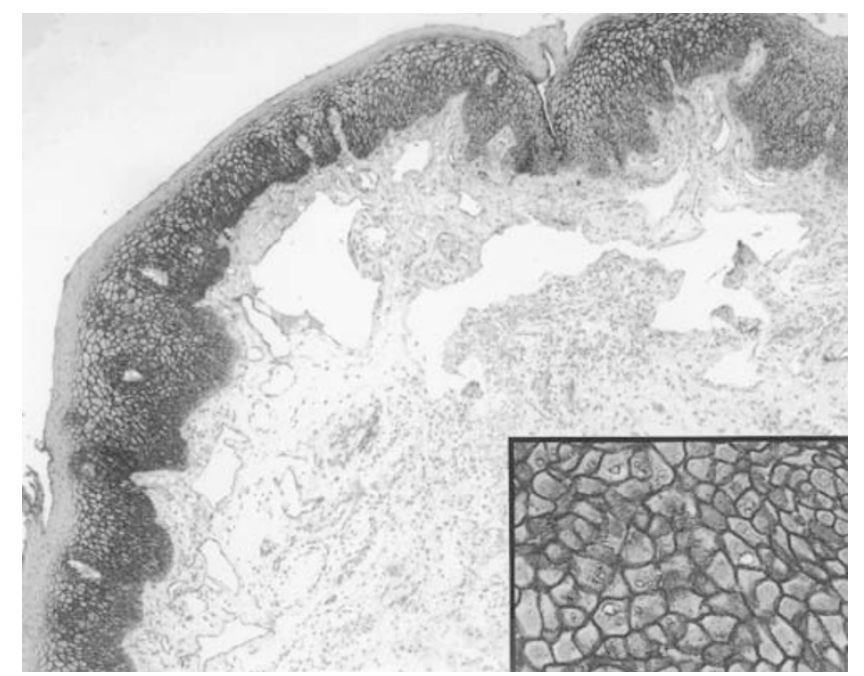

Figure I Tissue from normal laryngeal mucosae showed positive staining for $\mathrm{CD}$ 9 using $\mathrm{IHC}$ in the squamous epithelium, while the connective tissue is negative. The squamous epithelial cells are positive with membranous pattern (inset). 
regional disease (five regional only) and 19 with distant metastases (16 distant only). At 5 years, actuarial DFS was $58 \%$ (95\% CI $0.49-$ 0.67 ) and overall survival was $38 \%$ (95\% CI $0.30-0.46)$.

\section{CD9 expression and clinicopathologic associations}

On CD9 evaluation, 108 cases were CD9 positive with any staining pattern (cytoplasmic/membranous) and 45 showed reduced expression. Of the 108 positive cases, 85 had membranous staining and 23 had both membranous and cytoplasmic staining (Figures 2, 3). CD9 are expressed in 100, 70 and $10 \%$ of G1, G2 and G3 cases, respectively. When the CD9 expression was compared with various clinical features, there was no significant association between the CD9 expression and $\mathrm{T}$ stage $(P=0.41)$, lymph node status $(P=0.53)$, UICC stage grouping system $(0.77)$ or tumour sublocation $(P=0.3)$. However, there was a highly significant association between CD9 expression and tumour grade $(P=0.0007)$.

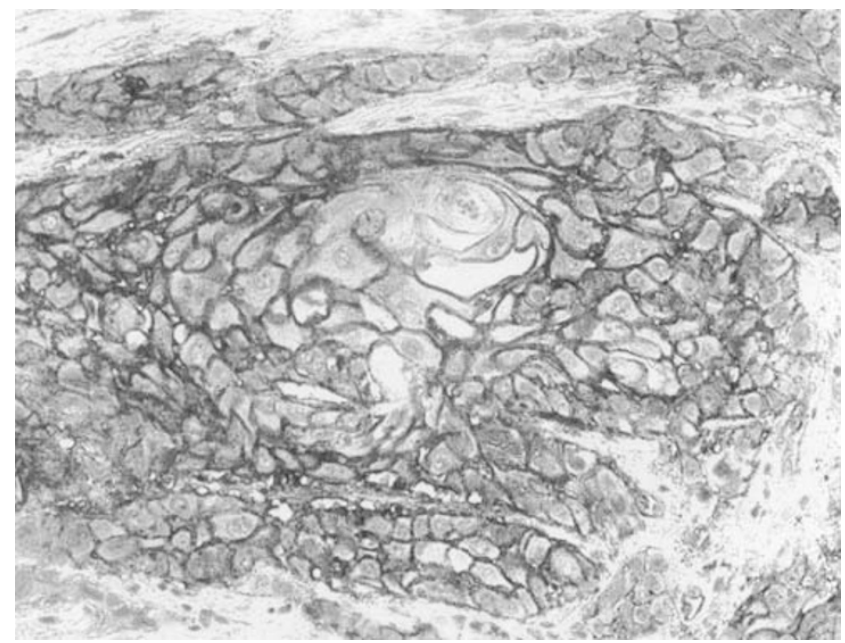

Figure 2 An example of positive staining for CD9, where $90 \%$ of tumour cells showed a strong reactivity with a membranous pattern.

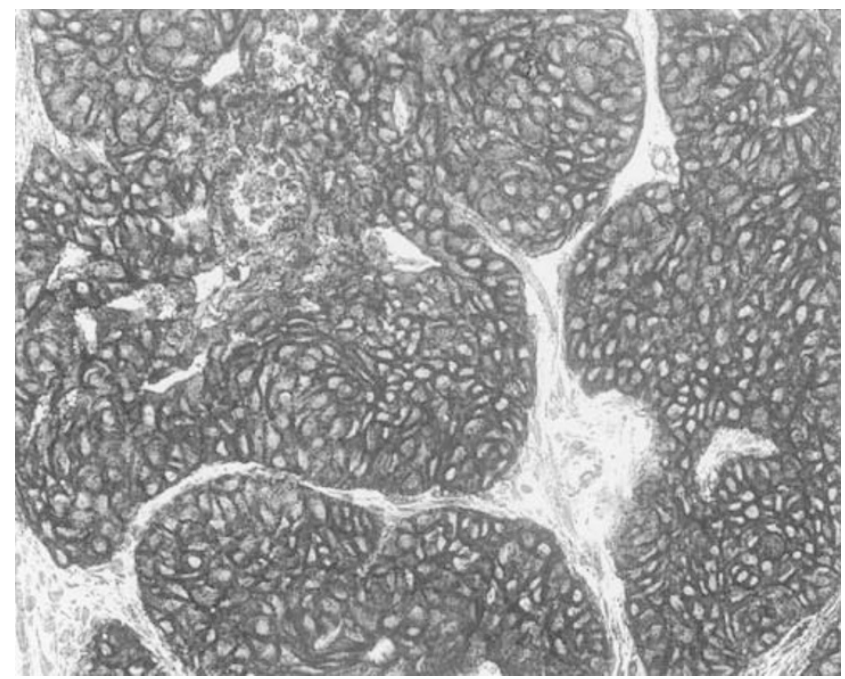

Figure 3 An example of the combined pattern where $>90 \%$ of tumour cells showed CD9 expression in both membranous and cytoplasmic patterns.

\section{Univariate and multivariate analyses}

In the univariate analysis, besides advanced $\mathrm{T}(P=0.013)$ and $\mathrm{N}$ categories $(P=0.002)$ and UICC stage $(P=0.002)$, the reduced expression of CD9 was significantly associated with lower 5-year DFS (43\% vs 64\%, $P=0.018$ ) (Figure 4). Histology grading (G1 vs G2-3) (Figure 5) and treatment category (with or without chemotherapy) did not correlate significantly with DFS.

Factors significantly influencing DFS in univariate analysis were included in the Cox model (except linked variables). In such a model, T-category $(P=0.024), \mathrm{N}$-category $(P=0.012)$ and CD9 expression $(P=0.017)$ retained their significance for DFS, and can consequently be considered as independent factors in predicting tumour failure. The relative risks associated with these factors are listed in Table 2. In a similar model, when substituting CD9 expression by tumour grading (G1 vs $\mathrm{G} 2-3$ ), the impact of the latter on DFS remained nonsignificant $(P=0.3)$.

\section{Subgroup analysis}

By contrast to tumours expressing only membranous CD9, the 23 tumours with both membranous and cytoplasmic staining showed

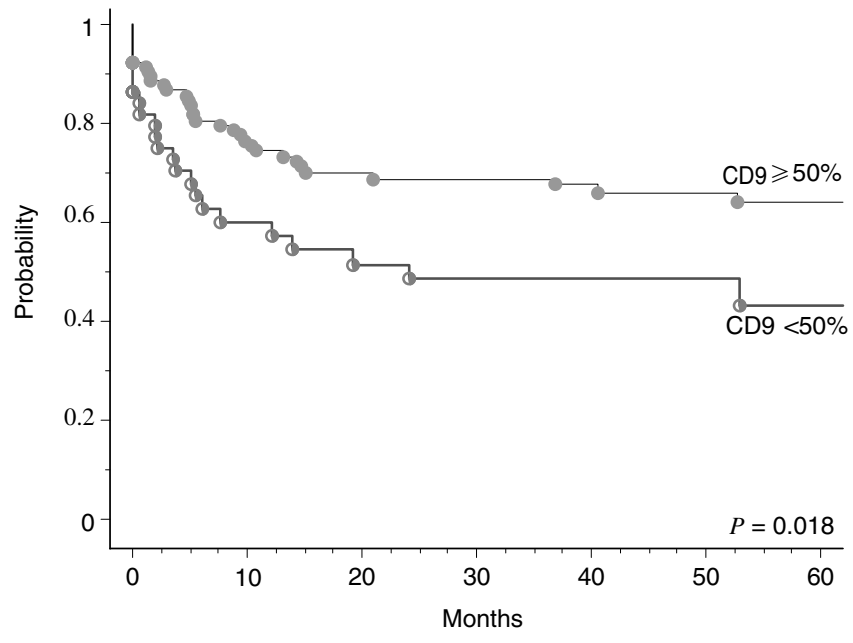

Figure 4 Actuarial DFS after therapy according to CD9 expression (all staining patterns (log-rank test of 0.018$)$ )

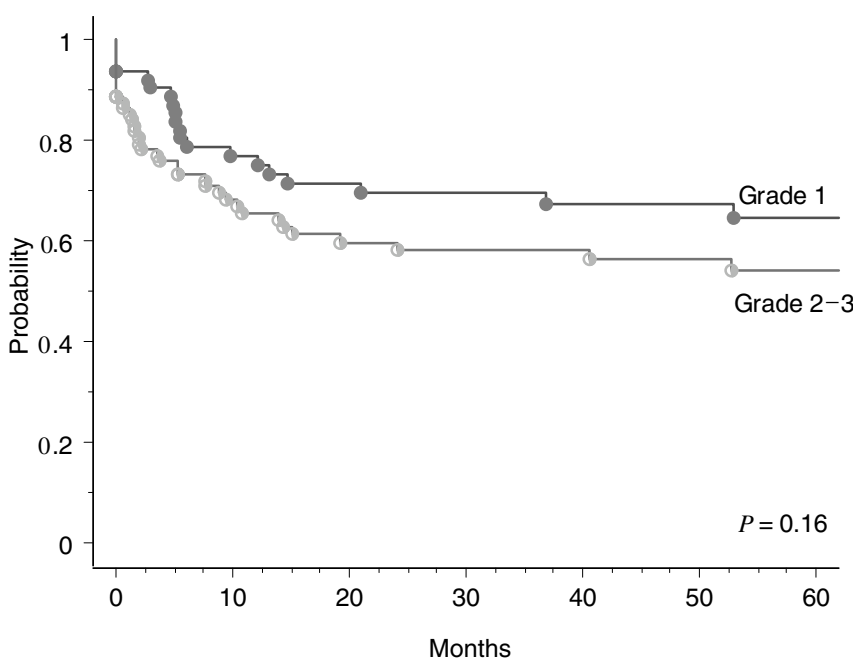

Figure 5 Actuarial DFS according to the histological grading (GI vs G23) (log-rank test 0.16). 
Table 2 Cox proportional hazards model for DFS

\begin{tabular}{llll}
\hline Variables & RR & $\mathbf{9 5 \%} \mathbf{C l}$ & P-value \\
\hline T -category: $\mathrm{TI}-2 / \mathrm{T} 3-4$ & 0.51 & $0.29-0.91$ & 0.024 \\
$\mathrm{~N}$-category: N0/NI-3 & 0.48 & $0.27-0.85$ & 0.012 \\
CD9 expression: $<50 \% / \geqslant 50 \%$ & 1.9 & $1.12-3.33$ & 0.017 \\
\hline
\end{tabular}

$\mathrm{DFS}=$ disease-free survival; $\mathrm{RR}=$ relative risk; $\mathrm{Cl}=$ confidence interval.

a trend toward significant association with tumour stage $(P=0.08)$ $(95 \%$ CI $3.5-86)$ and disease failure $(P=0.083)(95 \%$ CI $-1.7-57)$, while the association with tumour grade (G1 vs G2-3) was less strong $(P=0.025$ vs 0.0006$)$ (95\% CIs $11-68$ vs $10-37)$. Thus, in this subgroup (membranous and cytoplasmic staining), the mean tumour CD9 values in patients with and without any oncological event were 37 and $65 \%$, respectively $(P=0.08)$. The impact of CD9 expression on the 5-year actuarial DFS was more significant in the subgroup with both membranous and cytoplasmic pattern compared to the subgroup with membranous pattern only $(P=0.009$ vs 0.04$)$.

\section{DISCUSSION}

The present study of 153 patients with a long-term follow-up is the first to investigate the value of CD9 protein in SCC of the head and neck. Among all parameters analysed, such as tumour grade, location, age, lymph node status, UICC stage classification, tumour grade was the only parameter to show a significant association with CD9 expression: low grade tumours appeared to express the CD9 protein more frequently. On the other hand, the present study indicates that reduced CD9 expression by tumour cells seems to predict disease progression after RT with or without chemotherapy. The link between reduced CD9 expression and tumour failure was independent of tumour $\mathrm{T}$ and $\mathrm{N}$-categories, as found in the multivariate analysis. If CD9 is to be considered for clinical used in the future, that might add an additional 18 pound sterling to the patient's bill (which is an almost insignificant cost).

The expression of CD9 using the IHC technique has been identified in numerous solid tumours. By evaluating CD9 expression in oesophageal SCC, Uchida et al found that the 5year survival rate of patients with reduced CD9 is worser than those with positive CD9 expression (Uchida et al, 1999). Miyake et al (1996) found that reduced CD9 expression is strongly associated with a high frequency of metastatic lymph node in breast cancer patients, and might be used to identify patients at high risk for disease recurrence. Huang et al (1998) also showed the prognostic value of CD9 in patients with breast cancer. In their study, CD9-positive expression predicted a better rate of DFS. Higashiyama et al (1997) showed an inverse relation between CD9

\section{REFERENCES}

Allal AS, de Pree C, Dulguerov P, Bieri S, Maire D, Kurtz JM (1999) Avoidance of treatment interruption: an unrecognized benefit of accelerated radiotherapy in oropharyngeal carcinomas? Int J Radiat Oncol Biol Phys 45: 41-45

Crissman JD, Liu WY, Gluckman JL, Cummings G (1984) Prognostic value of histopathologic parameters in squamous cell carcinoma of the oropharynx. Cancer 54: 2995-3001

DeStefani A, Magnano M, Bussi M, Cravero L, Lerda W, Usai A, Cavalot A, Ragona R, Gabriele P, Valente G, Cortesina G (1997) Identification of clinical, biological and prognostic factors in recurring squamous cell carcinoma of the head and neck. Acta Otorhinolaryngol Ital 17: $219-224$ expression and DFS in patients with lung adenocarcinoma. In all these studies, CD9 expression was seen as a membranous pattern. However, there are two investigations in the literature describing both the cytoplasmic and membranous patterns, as noted in our study in addition to the usual membranous staining. The first was by Kawashima et al (2002) and the second was by Houle et al (2002)). In the former, CD9 expression was evaluated in different types of brain tumours, and they concluded that CD9 expression in astrocytic tumours correlated with their malignancy and thus CD9 protein may have a different role in brain tumours than solid tumours elsewhere. However, the cytoplasmic staining was not commented on. The latter evaluated CD9 expression in ovarian carcinoma. Loss of CD9 was found to be associated with tumour grade and, in particular, cytoplasmic CD9 expression with higher tumour grade. However, the study consisted of a small number of cases (38 cases), and the cytoplasmic staining was seen in a very few cases and very focally. Thus, the authors themselves did not reach any conclusion on the significance of their finding due to the size of the sample analysed. In addition, the cutoff value for positivity was not mentioned. In our study, we find that the impact of CD9 expression on DFS was more significant in the subgroup with both membranous and cytoplasmic patterns, compared to the subgroup with membranous pattern only. In addition, besides its association to tumour grade, there was an association with another prognostic parameter, tumour stage. However, due to the small sample size, we are not able to assess the true significance of this finding and we cannot really draw an accurate conclusion. Studies with larger samples are needed to clarify the true meaning of this finding. The only study with results directly comparable to ours is that by Kusukawa et al, who found a strong association between loss of CD9 expression and high incidence of lymph node metastasis and poor prognosis of patients with SCC of the oral cavity. However, in this study, the cutoff value for positive tumour was not clearly stated, and multivariate study using the Cox regression analysis was not done.

The ability of neoplastic cells to invade the surrounding tissue and metastasis to distant organs comes from the disruption of cell adhesion and alteration of cell motility. CD9, which plays an important role in cell growth, cell adhesion and motility, seems to be an ideal candidate. In this study, we showed that CD9 expression in SCC of the head and neck may be an independent factor in predicting DFS, and consequently it may have a potential use in identifying patients with higher risk of recurrence after therapy.

\section{ACKNOWLEDGEMENTS}

We would like to thank the histopathology laboratory, especially Mrs Danielle Fontana for her technical assistance, the immunohistochemistry laboratory for their help and Mr Ludovic Metral for his technical skill.
Grabenbauer GG, Muhlfriedel C, Rodel F, Niedobitek G, Horung J, Rodel C Martus P, Iro H, Kirchner T, Steininger H, Sauer R, Weidenbecher M, Distel L (2000) Squamous cell carcinoma of the oropharynx: ki-67 and p53 can identify patients at high risk for local recurrence after surgery and postoperative radiotherapy. Int J Radiat Oncol Biol Phys 48: 1041 1050

Hemer ME, Mannion BA, Berditchevski F (1996) Association of TM4SF proteins with integrins: relevance to cancer. Biochim Biophys Acta 1297: $67-71$

Higashiyama M, Doi O, Kodama K, Yokouchi H, Adachi M, Huang CL, Taki T, Kasugai T, Ishiguro S, Nakamori S, Miyake M (1997) Immunohistochemically detected expression of motility-related protein-1 (MRP-1/ 
CD9) in lung adenocarcinoma and its relation to prognosis. Int J Cancer 74: $205-211$

Houle CD, Ding X-Y, Foley JF, Afshari CA, Barrett JC, Davis BJ (2002) Loss of expression and altered localization of KAI1 and CD9 protein are associated with epithelial ovarian cancer progression. 86: $69-78$

Huang C-I, Kohno N, Ogawa E, Adachi M, Taki T, Miyake M (1998) Correlation of reduction in MRP-1/CD9 and KA1/CD82 expression with recurrences in breast cancer patients. Am J Pathol 153: 973-983

Ikeyama S, Koyama M, Yamaoko M, Sasada R, Miyake M (1993) Supression of cell motility and metastasis with human motility-related protein (MRP-1/CD9) DNA. I Ex Med 177: 1231 - 1237

Kawashima M, Dohn-ura K, Mekada E, Fukui M, Iwaki T (2002) CD9 expression in solid non-neuroepithelial tumors and infiltrative astrocytic tumors. J Histochem Cytochem 50: 1195-1203

Khademi B, Shirazi FM, Vasei M, Doroudchi M, Gandomi B, Modjtahedi H, Pezeshki AM, Ghaderi A (2002) The expression of p53, c-erbB-1 and cerbB-2 molecules and their correlation with prognostic markers in patients with head and neck tumors. Cancer Lett 184: 223-230

Khan AJ, King BL, Smith BD, Smith GL, DiGiovanna MP, Carter D, Haffty BG (2002) Characterization of the HER-2/neu oncogene by immunohistochemical and fluorescence in situ hybridization analysis in oral and oropharyngeal squamous cell carcinoma. Clin Cancer Res 8: $540-548$

Kusukawa J, Ryu F, Kameyama T, Mekada E (2001) Reduced expression of CD9 in oral squamous cell carcinoma: CD9 expression inversely related to high prevalence of lymph mode metastasis. J Oral Pathol Med 30: $73-79$

Landis SH, Murray T, Bolden S, Wingo PA (1999) Cancer statistics. CA Cancer I Clin 49: 8-31
Liu M, lawson G, Delos M, Jamart J, Chatelain B, Remacle M, Marbaix E (2003) Prognostic value of cell proliferation markers, tumour suppressor proteins and cell adhesion molecules in primary squamous cell carcinoma of the larynx and hypopharynx. Eur Arch Otorhinolaryngol 260: $28-34$

Maecker HT, Todd SC, Levy S (1997) The tetraspanin family: molecular facilitators. FASEB J 11: 428-442

Miyake M, Koyoma M, Seno M, Ikeyama S (1991) Identification of the motility-related protein (MRP-1), recognized by monoclonal antibody M31-15, which inhibits cell motility. J Exp Med 174: 1347-1354

Miyake M, Nakano K, Itoi S-I, Koh T, Taki T (1996) Motility-related protein-1 (MRP-1/CD9) reduction as a factor of poor prognosis in breast cancer. Cancer Res 56: $1244-1249$

Pera E, Moreno A, Galindo L (1986) Prognostic factors in laryngeal cancer: a multifactorial study of 416 cases. Cancer 58: $928-934$

Pukkila MJ, Virtaniemi JA, Kumpulainen EJ, Pirinen RT, Johansson RT, Valtonen HJ, Juhola MT, Kosma V-M (2001) Nuclear beta catenin expression is related to unfavourable outcome in oropharyngeal and hypopharyngeal squamous cell carcinoma. J Clin Pathol 54: $42-47$

Schoelch ML, Regezi JA, Dekker NP, Ng IOL, McMillan A, Ziober BL, Le QT, Silverman S, Fu KK (1999) Cell cycle proteins and the development of oral squamous cell carcinoma. Oral Oncol 35: 33-342

Uchida S, Shimada Y, Watanabe G, Li ZG, Hong T, Miyake M, Imamura M (1999) Motility-related protein (MRP-1/CD9) and KAI1/CD82 expression inversely correlate with lymph node metastsis in oesophageal squamous cell carcinoma. $\mathrm{Br} J$ Cancer 79: $1168-1173$

Wiernik G, Millard PR, Haybittle JL (1991) The predictive value of histological classification into degrees of differentiation of squamous cell carcinoma of the larynx and hypopharynx compared with the survival of pateints. Histopathology 19: $411-417$ 